\title{
Effect of processing on the structures and properties of bismuth sodium titanate compounds
}

\author{
Amit Mahajan, ${ }^{1, a)}$, Hangfeng Zhang ${ }^{1, a)}$ (B), Jiyue Wu ${ }^{1}$, E. Venkata Ramana ${ }^{2}$, Chuying Yu1 ${ }^{1}$, \\ Nadezda V. Tarakina ${ }^{1,3}$, M. J. Reece ${ }^{1}$, Haixue Yan $^{1}$ \\ ${ }^{1}$ School of Engineering \& Materials Science, Queen Mary University of London, Mile End Road, London E1 4NS, UK \\ ${ }^{2}$ I3N-Aveiro, Department of Physics, University of Aveiro, 3810193 Aveiro, Portugal \\ ${ }^{3}$ Max Planck Institute of Colloids and Interfaces, Colloid Chemistry, Research Campus Golm, Am Mühlenberg 1, 14476 Potsdam, Germany \\ a) Address all correspondence to these authors. e-mails: amahajan2@gmail.com; Hangfeng.zhang@qmul.ac.uk \\ Received: 28 August 2020; accepted: 30 November 2020; published online: 13 January 2021
}

The processing of sodium ( $\mathrm{Na}$ ) and bismuth (Bi)-based piezoelectric compounds are always a concern due to their volatilization at high temperatures $\left(>1000^{\circ} \mathrm{C}\right)$ and the hygroscopic nature of the precursors. The effect of drying and impurities in the precursors on $0.94\left(\mathrm{Bi}_{0.5} \mathrm{Na}_{0.5} \mathrm{TiO}_{3}\right)-0.06 \mathrm{BaTiO}_{3}(\mathrm{BNTBT})$ ceramics was investigated. A substantial difference in the weight fraction of polar (R3C) and weak-polar (P4bm) phase at room temperature was observed for BNTBT obtained from dried, undried and low purity precursors, evaluated by XRD and Raman. The local crystal structure was evaluated by transmission electron microscope, which showed the presence of both ferroelectric domains and polar nano regions. The electrical measurements corroborated well with the structure results, and showed a variation in the depolarization temperature $\left(T_{\mathrm{d}}\right)$. The results highlight the importance of the pre-processing steps on the functional compounds obtained from sensitive elements, which has broader implementations for similar systems.

\section{Introduction}

Lead-based compounds (such as $\mathrm{PbZr}_{1-x} \mathrm{Ti}_{x} \mathrm{O}_{3}, \mathrm{PZT}$ ) are primarily used as piezoelectric actuators, sensors, transducers and other electronic devices [1]. However, the toxicity of leadbased compounds is a concern for the environment and living beings. RoHS (The Restriction of the Use of Certain Hazardous Substances) and WEEE (Waste Electrical and Electronic Equipment recycling) regulations have limited the use of hazardous elements in electronics, one among them is lead [2]. Therefore, lead free piezoelectric materials have been a major topic of scientific research since last two decades [3]. One of the promising lead free materials with high Curie temperature $\left(>250^{\circ} \mathrm{C}\right)$ and fairly good piezoelectric constant $\left(d_{33}>130 \mathrm{pC} / \mathrm{N}\right)$ is $\mathrm{Bi}_{0.5} \mathrm{Na}_{0.5} \mathrm{TiO}_{3}-\mathrm{BaTiO}_{3}$ (BNTBT) solid solutions [4]. The first work on BNTBT was published in 1991 by Takenaka et al. [4], where the authors studied the electrical properties and phase transitions of $(1-x)\left(\mathrm{Bi}_{0.5} \mathrm{Na}_{0.5} \mathrm{TiO}_{3}\right)-x\left(\mathrm{BaTiO}_{3}\right)$ compounds (where $x$ varied from 1 to $30 \mathrm{~mol} \%$ ). Based on the electrical measurements they established the morphotropic phase boundary (MPB) for BNTBT system lies close to $6 \mathrm{~mol}$. \% of $\mathrm{BaTiO}_{3}$, where it shows maximum dielectric constant $(\sim 1000)$, high piezoelectric constant $(125 \mathrm{pC} / \mathrm{N})$, a maximum permittivity at around $288^{\circ} \mathrm{C}$ and bending strength of $200 \mathrm{MPa}$. Following this work, a thorough investigation of the structure at MPB was carried out by neutron diffraction, X-ray powder diffraction (XRD), transmission electron microscopy (TEM), Raman spectroscopy and differential thermal calorimetry [5-9]. These studies illustrate the presence of both rhombohedral (polar) and tetragonal (weak polar) phase at room temperature up to $100{ }^{\circ} \mathrm{C}\left(T_{\mathrm{d}}\right.$, depolarization temperature), above which the tetragonal phase dominates. BNT exhibits long range ferroelectric ordering at room temperature [10]. The TEM studies on BNT crystal shows a complex pattern of ferroelectric domains with lamellar or needle shaped morphology, indicative of long range ordering at room temperature [11]. However, most of the TEM studies on BNTBT (with 6 mol\% of $\mathrm{BaTiO}_{3}$ ) show the 
presence of nano polar regions (up to few $\mathrm{nm}$ in size) without long range ferroelectric domains, which might be due to the weak polar phase for unpoled BNTBT at RT or lack of homogeneity in the samples due to the presence of sensitive elements $[8,12-14]$. Nevertheless, a comprehensive crystal structural investigation using in situ TEM for unpoled and poled BNTBT solid solution by Ma et al. $[12,13]$ concluded that BNT with $6 \mathrm{~mol} \%$ of BT consists of both R3c (polar) and P4bm (weak polar) phases at room temperature, and display an electric field induced $(>2.7 \mathrm{kV} / \mathrm{mm})$ phase transition to the polar phase $(P 4 \mathrm{~mm})$. However, the clarification of the crystal structure of $\mathrm{BNTBT}$ and the base compound BNT at room temperature is still open for discussion; this is due to reports of the existences of monoclinic or co-existence of orthorhombic and rhombohedral phases [6,15-18]. The dielectric parameters are highly sensitive to the crystal structural and extrinsic parameters, which could lead to the variation in the dielectric parameters such as depolarization temperature $\left(T_{\mathrm{d}}\right)$, shoulder temperature $\left(T_{\mathrm{s}}\right)$, maximum temperature $\left(T_{\mathrm{m}}\right)$ and the dispersion in the dielectric permittivity as a function of temperature for $0.94(\mathrm{Bi}$ ${ }_{0.5} \mathrm{Na}_{0.5} \mathrm{TiO}_{3}$ )-0.06 $\mathrm{BaTiO}_{3}$ (BNTBT) published elsewhere (discussed later) $[14,19,20]$.

BNTBT is often prepared using sensitive precursors, $\mathrm{Na}_{2} \mathrm{CO}_{3}$ and $\mathrm{Bi}_{2} \mathrm{O}_{3}$, which are hygroscopic and volatile at high temperature due to their low melting temperature [21]. These issues could result in off-stoichiometry and/or lack of coherence in the BNTBT lattice at the A-site ( $\mathrm{Na}$ and $\mathrm{Bi}$ ions) which could result in the variation of coherent length of the octahedral tilting and result in modulation of the structural and electrical properties. Therefore, it is important to investigate how the deficiency of the A-site ions ( $\mathrm{Na}$ and $\mathrm{Bi}$ ) and the effect of secondary ions from low purity precursors on the properties of BNTBT compounds. There have been studies on the effect of off-stoichiometry in which there was a deliberate attempt to study the effect of excess or deficient of A site ions in $0.94\left(\mathrm{Bi}_{0.5} \mathrm{Na}_{0.5} \mathrm{TiO}_{3}\right)-0.06 \mathrm{BaTiO}_{3}(\mathrm{BNTBT})$ on its electrical properties. However, these studies did not mention the effect of stoichiometry on any structural changes $[22,23]$. A study on the base compound BNT shows that the off stoichiometry of Bi and $\mathrm{Na}$ ion in the BNT system tuned the BNT from insulator to ionic conductor [24-26]. Nevertheless, there are no studies related to the effect of non-stoichiometry due to hygroscopic nature and the use of low purity precursors on the structural and electrical properties. Therefore, in the present work we have highlighted a very relevant concern on the processing of sensitive compounds based on $\mathrm{Na}$ and $\mathrm{Bi}$. We systematically studied the effect of preheating of the precursors and effect of purity of the precursor on the highly disorder BNTBT (6 mol\% of BNT in BNT) system. This study has a wider consequence for other related ferroelectric compounds containing $\mathrm{Na}$ and Bi ions.

\section{Results and discussion}

Both the sodium $\left(\mathrm{Na}_{2} \mathrm{CO}_{3}\right)$ and bismuth $\left(\mathrm{Bi}_{2} \mathrm{O}_{3}\right)$ precursors are hygroscopic in nature. In order to investigate the kinetics of water adsorption from the atmosphere, the $\mathrm{Na}$ and Bi precursors were heat treated at $400{ }^{\circ} \mathrm{C}$ for $24 \mathrm{~h}$ and dried in a desiccator. As soon as the temperature reached close to the room temperature the precursors were put into a glass bottle, covered with alumina foil, and the initial weight of the dried precursors were obtained $\left(\mathrm{Na}_{2} \mathrm{CO}_{3}=23.082 \mathrm{~g}, \mathrm{Bi}_{2} \mathrm{O}_{3}=29.2339 \mathrm{~g}\right)$. The average temperature and relative humidity in the atmosphere were $15^{\circ} \mathrm{C}$ and $86 \%$, respectively. The weight of both precursors was measured as the function of time (Fig. 1). In the case of $\mathrm{Bi}_{2} \mathrm{O}_{3}$ precursor, the maximum weight change was less than $0.0031 \mathrm{~g}(0.01 \%)$ after $214 \mathrm{~h}$ (more than 8 days). However, the maximum weight gain $(0.0026 \mathrm{~g}, 0.009 \%)$ occurred in the initial $9 \mathrm{~h}$, which is 2 times more than the error associated with the measurements. This indicates that the Bi precursor reached its maximum water adsorption (if any) from the surroundings within the initial few hours and might not be significantly hygroscopic in nature. However, in the case of $\mathrm{Na}_{2} \mathrm{CO}_{3}$ there was a linear increase in the weight gain; with an initial weight gain within the first $24 \mathrm{~h}$ of more than $0.005 \mathrm{~g}(0.02 \%)$ and after $214 \mathrm{~h}$ a weight gain of $0.0232 \mathrm{~g}(0.1 \%)$. This would lead to a significant error in the composition of the final compounds if the precursors are not preheated before weighing according to the stoichiometry, especially for the sodium ions. To understand the effect of preheating of the precursor the ud-BNTBT, d-BNTBT and lp-BNTBT ceramics were prepared and their structural and electrical properties were investigated.

The crystal structure and phase distribution in the udBNTBT, d-BNTBT and lp-BNTBT were evaluated by XRD. The Bragg reflections from all three BNTBT compositions matches well with the pseudo-cubic BNT phase (JCPDS \# 01-3109) [27] and there was no formation of secondary phases, within the resolution limit of XRD (Fig. S1a). The extended pattern from pseudo-cubic (pc) plane (110) $)_{\mathrm{pc}}$ for d-BNTBT, ud-BNTBT and

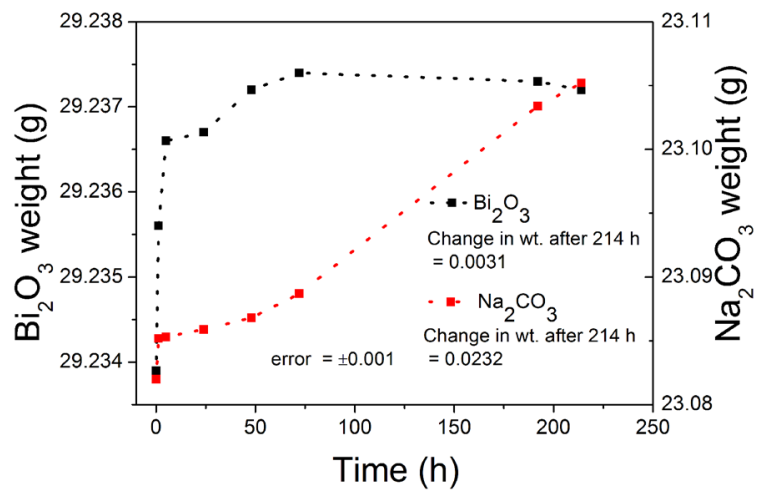

Figure 1: Illustration of the weight gained by sodium and bismuth oxides as a function of time due to hygroscopic nature of the precursors. 
lp-BNTBT is given in Fig. S1b. The Braggs reflection peaks from the $(110)_{\mathrm{pc}}$ planes for all the BNTBT compositions show the presence of both rhombohedral and tetragonal phase. There was a shift in the $(110)_{\mathrm{pc}}$ peak towards to lower $2 \theta\left(32.57^{\circ}\right)$ for lpBNTBT compared to d-BNTBT and ud-BNTBT $\left(32.60^{\circ}\right)$. The shift in diffraction peaks for lp-BNTBT is an indication of crystallochemical distortion which might be the result of the ions from impurities [28]. In order to draw a comprehensive analysis of the XRD results, Rietveld refinement was performed using GSAS software [29] to quantify the polar and weak polar phases (Fig. S2). Both rhombohedral (R3c) and tetragonal (P4bm) phases was present for all the BNTBT system at room temperature (Table 1). The ud-BNTBT, d-BNTBT and lp-BNTBT have weight fraction of $R 3 c$ (polar) phase around 56\%, 15\% and $28 \%$ and $P 4 \mathrm{bm}$ (weak polar) phase of $44 \%, 85 \%$ and $72 \%$, respectively. The lower percentage of $R 3 c$ phase for ud-BNTBT (15\%) in comparison to d-BNTBT (56\%) is most probably due to the deficiency of the A site cations $(\mathrm{Na}, \mathrm{Bi})$, which results in the creation of oxygen vacancies and A site vacancies in the BNTBT lattice, represented by Kroger-Vink equations (Eqs. 1, 2). Consequently, the deficiency at the A site in BNTBT results in the distortion in oxygen octahedra that leads to the variation of the $R 3 c$ and $P 4 b m$ phase distribution in d-BNTBT and ud-BNTBT $[11,24]$.

$$
\begin{aligned}
& 2 \mathrm{Na}_{\mathrm{Na}}+\mathrm{O}_{\mathrm{o}} \rightarrow 2 \mathrm{~V}_{\mathrm{Na}}^{\prime}+\mathrm{V}_{\ddot{o}}+\mathrm{Na}_{2} \mathrm{O}(\mathrm{g}), \\
& 2 \mathrm{Bi}_{\mathrm{Bi}}+3 \mathrm{O}_{\mathrm{o}} \rightarrow 2 \mathrm{~V}_{\mathrm{Bi}}^{\prime \prime \prime}+3 \mathrm{~V}_{\ddot{\mathrm{o}}}+\mathrm{Bi}_{2} \mathrm{O}_{3}(\mathrm{~g}) .
\end{aligned}
$$

In the case of lp-BNTBT, the weight fraction of $R 3 c$ phase (28\%) was lower than for d-BNTBT, even though both these samples were prepared by initial heating of the precursors. This decrease can be associated with the ions from the impurities.

Based on group theory BNT with R3c space group has 13 Raman active modes $7 \mathrm{~A} 1+6 \mathrm{E}[7,30]$. The Raman active modes for BNT observed between 80 and $150 \mathrm{~cm}^{-1}$ are assigned to $\mathrm{A}-\mathrm{O}$ vibrations (where $A$ is cation), whereas the peaks at 260, 535, and $619 \mathrm{~cm}^{-1}$ are assigned to O-Ti-O vibrations [7]. The solid solution of $\mathrm{BNT}$ with $6 \mathrm{~mol} \%$ of $\mathrm{BaTiO}_{3}$ results in the splitting of the $260 \mathrm{~cm}^{-1}$ peak in to two broad peaks at $253 \mathrm{~cm}^{-1}$ and $312 \mathrm{~cm}^{-1}$ [30]. The peak at $312 \mathrm{~cm}^{-1}$ belongs to the $\mathrm{B} 1 / \mathrm{E}$ $(\mathrm{TO}+\mathrm{LO})$ mode, and is a fingerprint for the tetragonal phase [31]. The Raman peak positions and the full-width half maximum (FWHM) of the peaks are related to the rhombohedral and tetragonal structures. These were evaluated by fitting the Raman data using a Gaussian function for d-BNTBT, ud-BNTBT and lp-BNTBT as shown in Fig. 2. The peaks observed from bending and stretching of $\mathrm{A}-\mathrm{O}$ and $\mathrm{Ti}-\mathrm{O}$ bonds for all the samples match well with the earlier reports $[5,30]$. The ratio of FWHM that corresponds to the tetragonal and rhombohedral phase (at $\sim 312.5 \mathrm{~cm}^{-1}$ and at $\sim 253 \mathrm{~cm}^{-1}$, respectively) for d-BNTBT (1.16) is lower than ud-BNTBT (1.19). This reflects that the volume fraction of tetragonal phase is more for ud-BNTBT, supporting the XRD results. Moreover, the Raman shift to lower wavenumber by $1 \mathrm{~cm}^{-1}$ for ud-BNTBT $\left(311.5 \mathrm{~cm}^{-1}\right)$ compared to d-BNTBT $\left(312.5 \mathrm{~cm}^{-1}\right)$ is associated with distortion induced by oxygen vacancies. However, there is no significant shift in the $\mathrm{A}-\mathrm{O}$ vibration mode and stretching and $\mathrm{O}-\mathrm{Ti}-\mathrm{O}$ bending modes, except for a little suppression in the intensity of the peak at $611 \mathrm{~cm}^{-1}$ for ud-BNT, which might be associated with the modulation in the local environment due to octahedral distortion compared to d-BNTBT.

For lp-BNT there was a significant shift in the A-O peak towards a lower wavenumber $\left(134 \mathrm{~cm}^{-1}\right)$ in comparison to d-BNTBT and ud-BNTBT $\left(136 \mathrm{~cm}^{-1}\right)$. The shift in A-O mode towards lower wavenumber is an indication that a part of $\mathrm{Na}$ site was occupied by heavier elements (such as $\mathrm{Pb} 207.2 \mathrm{amu}$ ) from the low purity precursors. The shift and decrease in the

\begin{tabular}{|c|c|c|c|c|c|c|}
\hline Sample & $\begin{array}{l}\text { Unit cell parameters }(\AA) \\
\text { (Phase } 1, P 4 b m)\end{array}$ & $\begin{array}{c}\text { Unit cell parameters }(\AA) \\
\text { (Phase } 2, R 3 c)\end{array}$ & Weight fraction (\%) & Fitting parameter & $\begin{array}{l}\text { Depolarization } \\
\text { temperature, } T_{\mathrm{d}} \\
\left({ }^{\circ} \mathrm{C}\right)\end{array}$ & $\begin{array}{c}\text { Shoulder } \\
\text { temperature, } \\
T_{\mathrm{s}}\left({ }^{\circ} \mathrm{C}\right)\end{array}$ \\
\hline \multirow[t]{4}{*}{ d-BNTBT } & $\underline{a}=b=5.5030(6)$ & $a=b=5.5068(3)$ & $P 4 b m=44.0$ & $\chi^{2}=1.388$ & 102 & 143 \\
\hline & $c=3.9302(9)$ & $c=13.563(1)$ & & & & \\
\hline & $a=\beta=\gamma=90^{\circ}$ & $a=\beta=90^{\circ}, \gamma=120^{\circ}$ & $R 3 c=55.983$ & $w R_{\mathrm{p}}=0.1232$ & & \\
\hline & Volume $=119.02(2)\left(\AA^{3}\right)$ & Volume $=356.2(5)\left(\AA^{3}\right)$ & & $R_{\mathrm{p}}=0.0976$ & & \\
\hline \multirow[t]{4}{*}{ ud-BNTBT } & $a=b=5.5171(3)$ & $a=b=5.532(1)$ & $P 4 b m=84.87$ & $\chi^{2}=1.284$ & 93 & 134 \\
\hline & $c=3.898(3)$ & $c=13.469(8)$ & & & & \\
\hline & $a=\beta=\gamma=90^{\circ}$ & $a=\beta=90^{\circ}, \gamma=120^{\circ}$ & $R 3 c=15.13$ & $w R_{\mathrm{p}}=0.1493$ & & \\
\hline & Volume $=118.67(1)\left(\AA^{3}\right)$ & Volume $=357.0(1)\left(\AA^{3}\right)$ & & $R_{\mathrm{p}}=0.1163$ & & \\
\hline \multirow[t]{4}{*}{ Ip-BNTBT } & $a=b=5.5172(4)$ & $a=b=5.5258(5)$ & $P 4 b m=71.53$ & $\chi^{2}=1.372$ & 100 & 165 \\
\hline & $c=3.8987(5)$ & $c=13.472(2)$ & & & & \\
\hline & $a=\beta=\gamma=90^{\circ}$ & $a=\beta=90^{\circ}, \gamma=120^{\circ}$ & $R 3 c=28.466$ & $w R_{\mathrm{p}}=0.1457$ & & \\
\hline & Volume $=118.68(2)\left(\AA^{3}\right)$ & Volume $=356.25(5)\left(\AA^{3}\right)$ & & $R_{\mathrm{p}}=0.1141$ & & \\
\hline
\end{tabular}

TABLE 1: Crystal structure refinement of ud-BNTBT, d-BNTBT and Ip-BNTBT for tetragonal (P4bm) and rhombohedral (R3C) phase. 


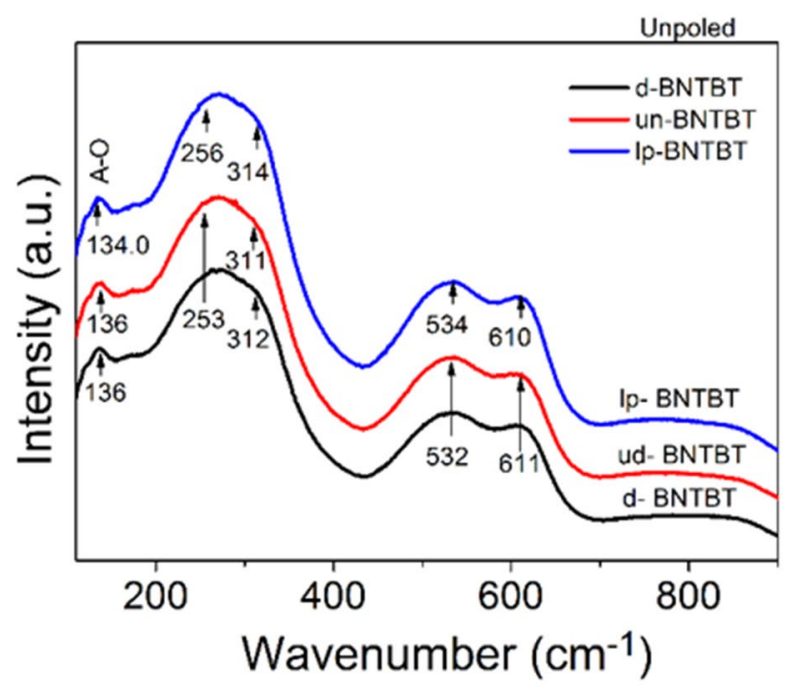

(a)

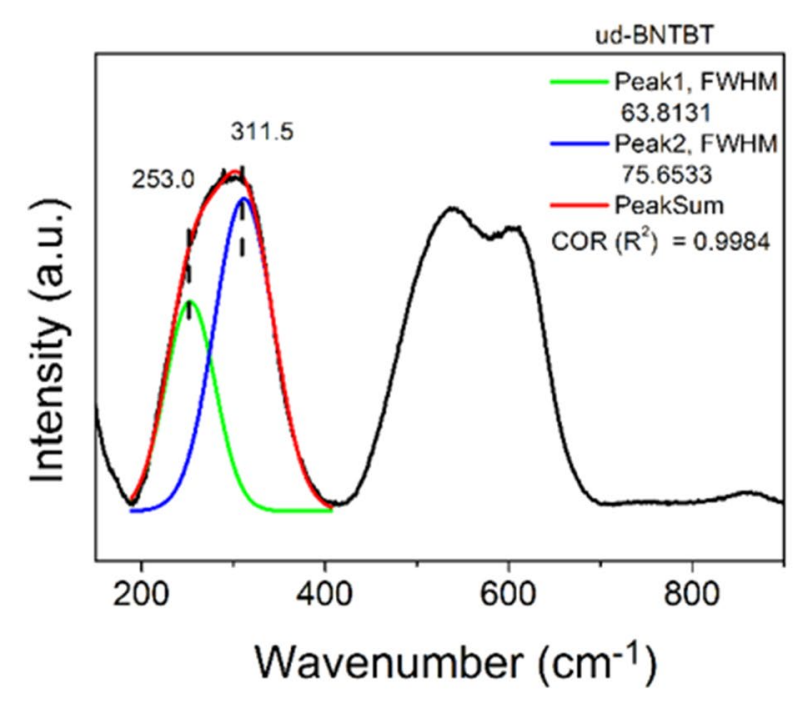

(c)
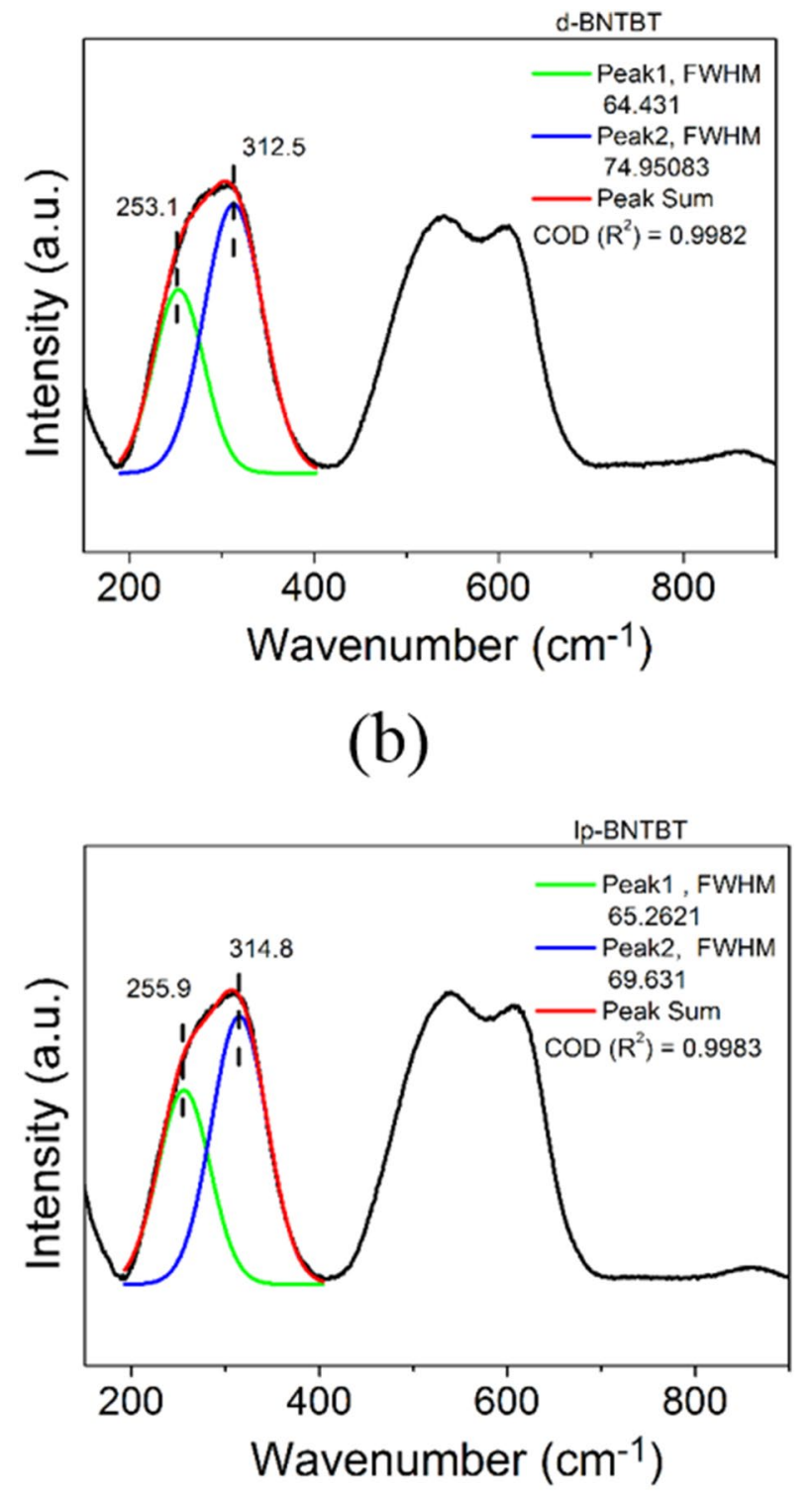

(d)

Figure 2: Raman spectra for d-BNTBT, ud-BNTBT and Ip-BNTBT (a), the Gaussian fitting of the O-Ti-O broad vibration band at $260 \mathrm{~cm}^{-1}$ for d-BNTBT (b), ud-BNTBT (c) and Ip-BNTBT (d). The higher ratio of FWHM for peak 2/peak 1 in case of ud-BNTBT as compared to d-BNTBT indicates that the ud-BNTBT is rich in weak polar phase (tetragonal) compared to d-BNTBT.

intensity of the $\mathrm{O}-\mathrm{Ti}-\mathrm{O}$ vibrational modes $\left(611 \mathrm{~cm}^{-1}\right)$ is associated with the tilting of the octahedra. This modulation in the local environment resulted in a significant variation in the phase distribution of the tetragonal and $R 3 c$ phases for d-BNTBT, udBNTBT and lp-BNTBT as observed by the XRD fitting. Overall, the vibration modes from all the three samples show typical characteristic bands with no additional bands, suggesting that overall structures are similar.

It was mentioned a few times that the variation of the $R 3 c$ and $P 4 \mathrm{bm}$ phases might be associated with the distribution of the $\mathrm{Na}$ and $\mathrm{Bi}$ atoms in the lattice $[11,32]$. In order to rationalize this statement, the elemental analysis was carried out on d-BNTBT and ud-BNTBT; SEM micrograph are shown in Fig. S3 and atomic and weight percentage are given in Tables S2 and $\mathrm{S} 3$, respectively. The ratio between the atomic percentage of $\mathrm{Bi}$ and $\mathrm{Na}$ ions taken from three different grains for d-BNTBT were 1.01, 0.99 and 0.97; and for ud-BNTBT 1.07, 1.02 and 1.07, respectively. In the case of $\mathrm{d}-\mathrm{BNTBT}$, the maximum difference between $\mathrm{Bi}$ and $\mathrm{Na}$ ions was $<0.34$ at.\% and negative, which illustrates that the main deficiency is of Bi ions, which might be 
due to volatilize of $\mathrm{Bi}$ and $\mathrm{Na}$ at high temperature [21]. Whereas, in the case of ud-BNTBT, the maximum difference between $\mathrm{Bi}$ and $\mathrm{Na}$ ions was $<0.65$ at. $\%$ and positive, indicates the grains are deficient in Na ions. This supports our kinetic results (Fig. 1) and concludes that off-stoichiometry (deficiency of $\mathrm{Na}$ ions) for udBNTBT due to the hygroscopic property of $\mathrm{Na}_{2} \mathrm{CO}_{3}$.

The local crystal structure and ferroelectric domains in unpoled d-BNTBT were studied using TEM (Figs. 3, S4). The low magnification TEM image from the d-BNTBT lamella (Fig. S4a) shows an area with a clear boundary between three grains of about $\sim 3 \mu \mathrm{m}$ in size. Figure $3 \mathrm{~b}, \mathrm{c}$ show selected area electron diffraction (SAED) patterns obtained from different grains. Indexing of patterns is done in tetragonal (white markers) and rhombohedral (green markers) unit cells obtained from XRD refinement, however, the presence of weak diffraction spots indicated by green arrows clearly shows that neither of these unit cells can describe the SAED patterns completely. The orthorhombic unit cell [sp.gr. Bmmb (63), $a=7.73 \AA$, $b=7.74 \AA, c=7.74 \AA$ ] allows full indexing of diffraction patterns (Fig. S5). The latter suggests that on the nanoscale the domain structure is rather close to a distorted cubic structure; the relations between tetragonal, rhombohedral and perovskite cubic lattices are shown on Fig. 3a. The diffraction spots marked by red squares in Fig. $3 \mathrm{~b}$ and $\mathrm{c}$, have been used to form dark field images on Fig. 3d and e, respectively. These dark-field images show a complex pattern of ferroelectric domains with herringbone microstructure very similar to the one seen in the parent compound $\mathrm{Na}_{0.5} \mathrm{Bi}_{0.5} \mathrm{TiO}_{3}$ (NBT) [11] and rhombohedral/tetragonal PZT [33]. Importantly, BNTBT investigated previously by TEM showed the presence of polar nano regions domains without any long range ordered ferroelectric domains for unpoled BNTBT $[12,14,34]$, and ferroelectric domains were only visible after dc poling, which induces long range ferroelectric ordering [14] or with high mol\% of BT (>10\%) where the crystal structure is dominated by the $P 4 \mathrm{~mm}$ space group (polar phase) [13]. It is worth to mention that several diffraction patterns were described in the rhombohedral lattice, suggesting that both rhombohedral and orthorhombic domains coexist at the nanoscale. Figure $3 f$, g show dark field images of d-BNTBT; the complex geometry of the ferroelectric domain as well as the presence of irregular domain walls, clearly visible on the images, are signatures of the $180^{\circ}$-domains [35]. Moreover, there are regions in the grains where the large ferroelectric domains are not present (marked with dotted line). The magnified view from such areas shows the presence of a knitted-like structure, which is associated with the polar nano domains $[12,14,34]$. Thus, we can conclude that in general the nanostructured features corroborate with findings from XRD and Raman measurements,
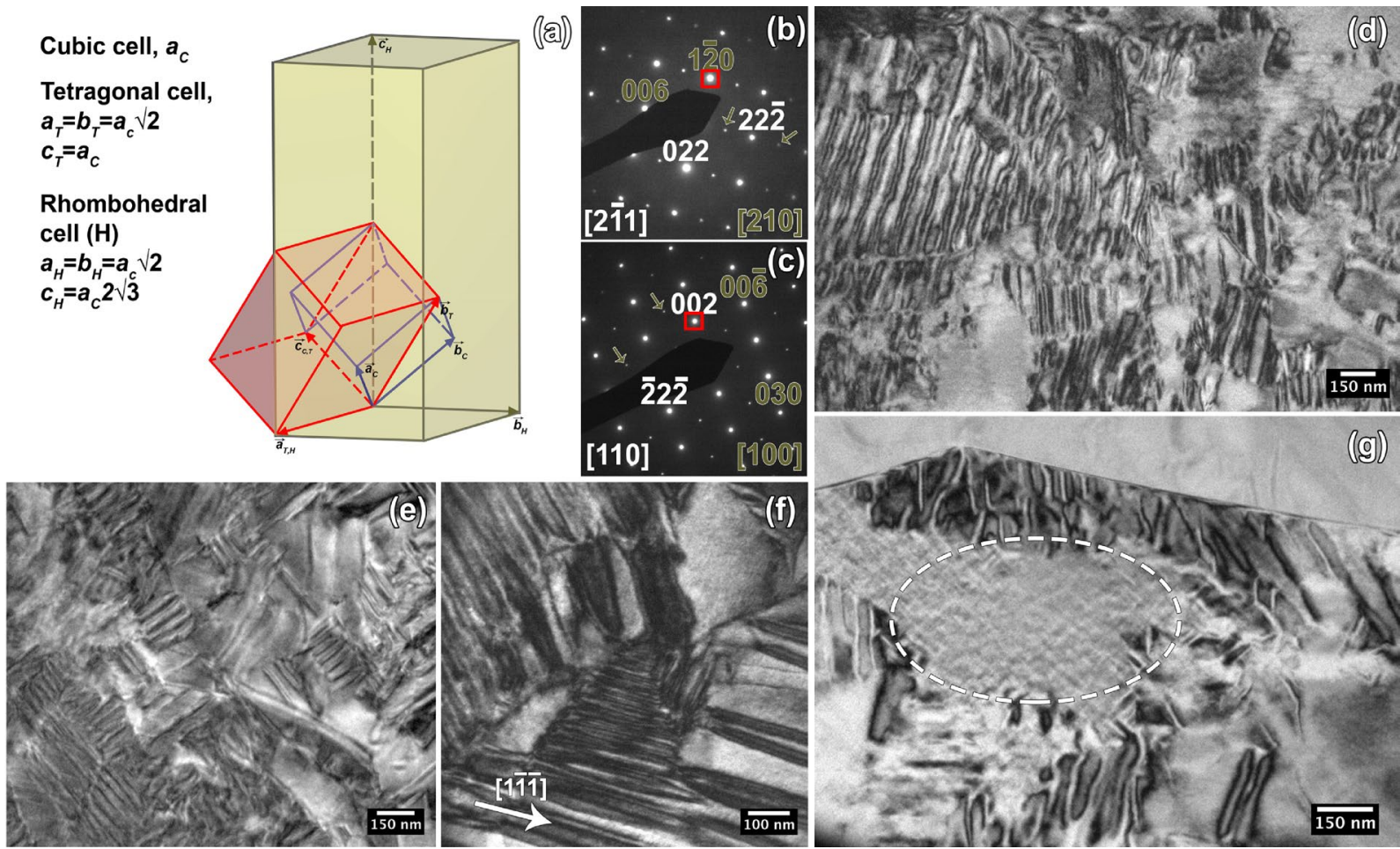

Figure 3: (a) Relation between perovskite cubic, tetrahedral and rhombohedral unit cells, (b, c) SAED patterns obtained from two different grains. White numbers denote indexing in the tetragonal unit cell, green numbers denote indexing in the rhombohedral unit cell. Red squares indicate diffraction spots from which dark field images on (d) and (e) were obtained. (f) and (g) Dark field images of the $180^{\circ}$ domain rotational domain with the irregular grain boundaries and the knitting structure of nano domains (marked by the dotted line). 
however, we were not able to prove the co-existence of the $R 3 \mathrm{c}$ and $P 4 \mathrm{bm}$ phases in the BNTBT system, but rather the presence of a complex interplay between rhombohedral and orthorhombic domains. Importantly, the presence of both long range ferroelectric domains and polar nano regions seen for BNTBT (with $6 \mathrm{~mol} \%$ of BT) is reported for the first time.

The electrical signatures are very sensitive to the structural changes, therefore the electrical properties were carried to study the effect of processing on all of the three compositions of BNTBT. The dielectric permittivity $\left(\varepsilon_{\mathrm{r}}\right)$ and loss tangent $(\tan \delta)$ as a function of frequency for d-BNTBT, ud-BNTBT and lp-BNTBT at room temperature are given in Fig. S6. The room temperature dielectric permittivity (at $1 \mathrm{kHz}$ ), loss tangent (at $1 \mathrm{kHz}$ ) and piezoelectric coefficient $\left(d_{33}\right)$ values are given in Table S4. d-BNTBT shows high dielectric constant (1280), lower losses (0.06) and higher $d_{33}(130 \mathrm{pC} / \mathrm{N})$ in comparison to the ud-BNT and lp-BNTBT.

The dielectric permittivity $\left(\varepsilon_{\mathrm{r}}\right)$ and loss tangent $(\tan \delta)$ were investigated as a function of temperature for unpoled (Fig. 4) and poled (Fig. 5) d-BNTBT, ud-BNTBT and lp-BNTBT. There were two/three anomalies present for unpoled/poled d-BNTBT,
ud-BNTBT and lp-BNTBT. The unpoled BNTBT (all three compositions) shows frequency dispersion in $\varepsilon_{\mathrm{r}}$ from RT up to $\sim 130{ }^{\circ} \mathrm{C}$, whereas the poling of BNTBT resulted in almost vanishing of the frequency dispersion in $\varepsilon_{\mathrm{r}}$ up to a certain temperature known as depolarization temperature $\left(T_{\mathrm{d}}\right)$ (first anomaly for poled BNTBT); above $T_{\mathrm{d}}$ the frequency dispersion in $\varepsilon_{\mathrm{r}}$ appears. The disappearance of the frequency dispersion below $T_{\mathrm{d}}$ for poled BNTBT is associated with long range ferroelectric ordering of the diffuse polar nano regions (PNR) produced by the external field. Correspondingly, a sharp anomaly was observed in $\tan \delta$ for poled BNTBT. The $T_{\mathrm{d}}$ temperature for d-BNTBT, ud-BNTBT and lp-BNTBT is $102{ }^{\circ} \mathrm{C}, 93^{\circ} \mathrm{C}$ and $100^{\circ} \mathrm{C}$, respectively. The modulation in the depolarization temperature is associated with the structural variation of the polar phases associated with the lattice distortion [36]. However, in the case of lp-BNTBT the shift in the $T_{\mathrm{d}}$ is associated with the secondary ions (Fe and $\mathrm{Pb}$ ) on the A-/B-sites of the BNTBT lattice. Above $T_{\mathrm{d}}$ the frequency dispersion appears for poled $\mathrm{BNTBT}$ due to a thermally driven phase transition from FE to relaxor phase. The second anomaly for poled BNTBT appears at $152{ }^{\circ} \mathrm{C}, 134{ }^{\circ} \mathrm{C}$ and $165^{\circ} \mathrm{C}$ for d-BNTBT, ud-BNTBT and

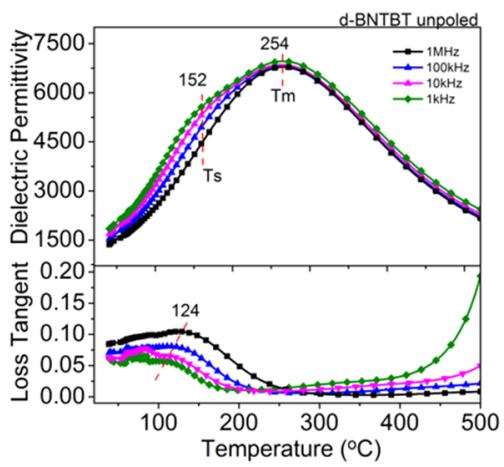

(a)

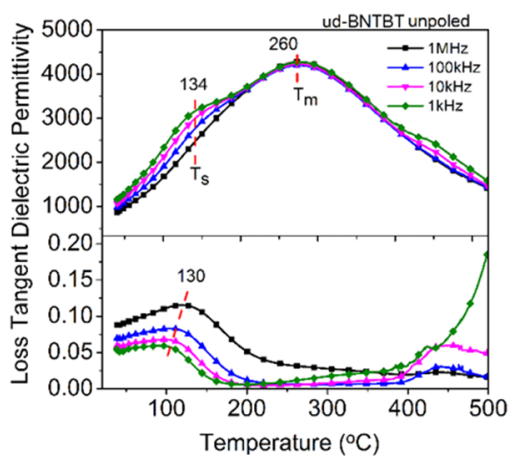

(b)

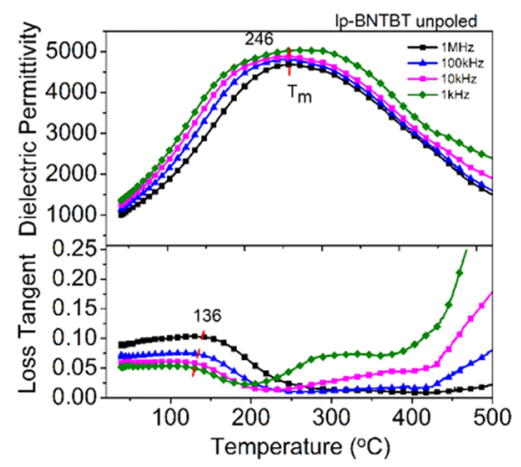

(c)

Figure 4: Dielectric permittivity and loss tangent as a function of temperature for unpoled (a) d-BNTBT, (b) ud-BNTBT and (c) Ip-BNTBT.

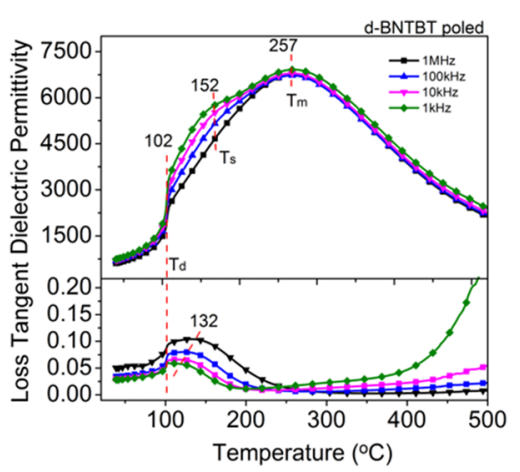

(a)

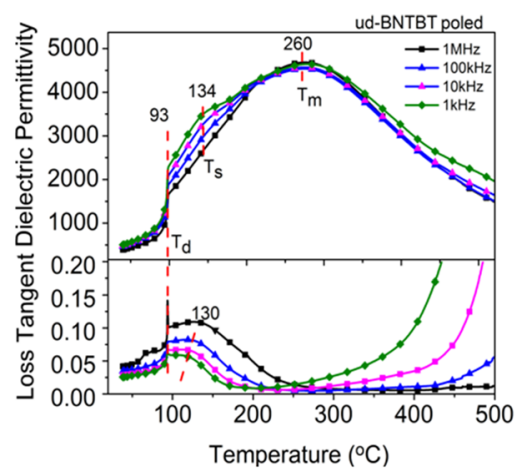

(b)

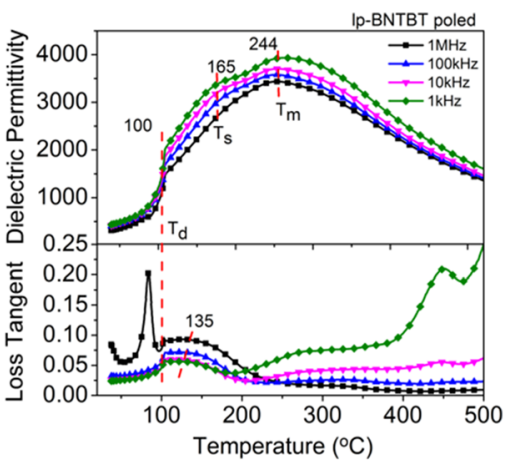

(c)

Figure 5: Dielectric permittivity and loss tangent as a function of temperature for poled (a) d-BNTBT, (b) ud-BNTBT and (c) Ip-BNTBT. 
lp-BNTBT (Fig. 5). Thereafter, the frequency dispersion starts to decrease and subsequently disappears at a point known as shoulder temperature $\left(T_{\mathrm{s}}\right)$. The Jo et al. [37] used multiple Debye type relaxations time function model to understand the relationship between the PNR with the $T_{s}$. Also, Viola et al. [38] used impedance spectroscopy to study the origin of $T_{\mathrm{s}}$ based on activation energy. Both studies conclude that there are two independent processes responsible for this anomaly with no specific rationalization. However, it is still open for detailed discussion. The diffuse transition is a consequence of a thermal evolution of the correlation length distribution of the PNR, and is fingerprint of relaxor behavior [37]. The third anomaly for poled BNTBT associated with the temperature where the BNTBT shows maximum dielectric permittivity is known as the maximum temperature $\left(T_{\mathrm{m}}\right)$. The $T_{\mathrm{m}}$ for poled d-BNTBT, ud-BNTBT and lp-BNTBT is $257^{\circ} \mathrm{C}, 260^{\circ} \mathrm{C}$ and $244^{\circ} \mathrm{C}$, respectively.

In the case of unpoled lp-BNTBT, the frequency dispersion in $\varepsilon_{\mathrm{r}}$ profile remains throughout the temperature range of the measurement, different to ud-BNTBT and d-BNTBT. The discrepancy in the dielectric profile for lp-BNTBT mainly relates to the presence of impurity ions. This can be substantiated by the Raman shift in the A-O mode and Ti-O modes observed for lp-BNTBT compared to d-BNTBT (Fig. 2).

Nonlinear polarization $(P)$-current $(I)$-electric field $(E)$ hysteresis loops as a function of temperature were obtained for d-BNTBT, ud-BNTBT and lp-BNTBT (Figs. 6, 7, 8), respectively. All the BNTBT exhibits typical ferroelectric loops with remnant polarization $\left(P_{\mathrm{r}}\right)$ of $38 \mu \mathrm{C} / \mathrm{cm}^{2}, 31 \mu \mathrm{C} / \mathrm{cm}^{2}$ and $35 \mu \mathrm{C} / \mathrm{cm}^{2}$ for d-BNTBT, ud-BNTBT and lp-BNTBT, respectively (Table S4).
The coercive field $\left(E_{\mathrm{c}}\right)$ values for d-BNTBT, ud-BNTBT and lpBNTBT, where domain switching take place, are $28.8 \mathrm{kV} / \mathrm{cm}$, $30 \mathrm{kV} / \mathrm{cm}$ and $33 \mathrm{kV} / \mathrm{cm}$, respectively (Table S4). The increase in the $E_{\mathrm{c}}$ for ud-BNTBT and lp-BNTBT is related to the pinning of the ferroelectric domains by the oxygen vacancies (in ud-BNTBT and lp-BNTBT). The oxygen vacancy in lp-BNTBT can be attributed to the B-site acceptor dopant of Fe which is from the impurity (Table $\mathrm{S} 1$ ).

On heating, there are four current peaks appearing in first and third quadrant in $I-E$ loops with high $P_{\mathrm{r}}$ in $P-E$ loops. This is associated with the field induced phase transition between polar and weak polar phase in BNTBT. Further increase in temperature, these four current peaks appeared at each quadrant of $I-E$ loops with low $P_{\mathrm{r}}$ in $P-E$ loops, which the field induced phase transition is recoverable after removal of electric field [39].

The electromechanical response $\left(d_{33}\right)$ for BNTBT was measured at RT after holding the samples at different temperatures. The normalized $d_{33}$ as a function of temperature is shown in Fig. 9. At RT the d-BNTBT shows high $d_{33}(130$ $\mathrm{pC} / \mathrm{N})$, followed by ud-BNTBT $(120 \mathrm{pC} / \mathrm{N})$ and lp-BNTBT $(90 \mathrm{pC} / \mathrm{N})$ (Table S4). Similar values for BNTBT have been reported in the literature $[4,40]$. However, a significant decrease in the $d_{33}$ for lp-BNTBT is associated with its low dielectric permittivity and high $E_{c}$. The variation of the $d_{33}$ can be related to the $R 3 c$ and $P 4 b m$ phase variation. As a function of temperature, almost stable $d_{33}$ were recorded up to $100{ }^{\circ} \mathrm{C}$ for all the three compositions. A sharp decrease in $d_{33}$ was observed for ud-BNTBT above $100{ }^{\circ} \mathrm{C}$ and for d-BNTBT above $120^{\circ} \mathrm{C}$. This observation is consistent with

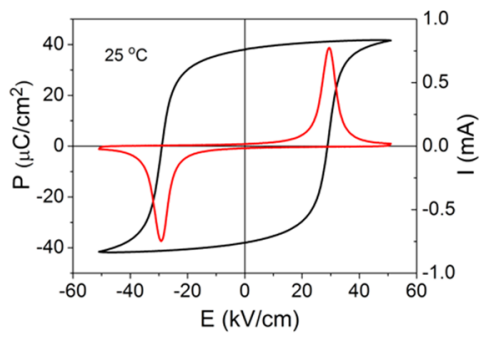

(a)

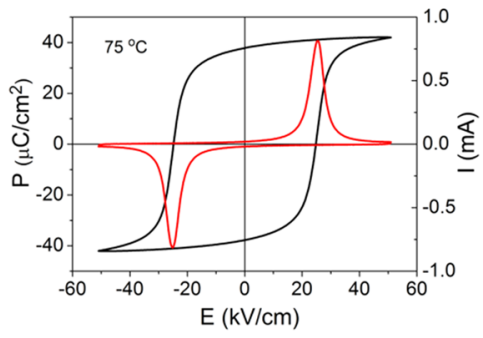

(b)

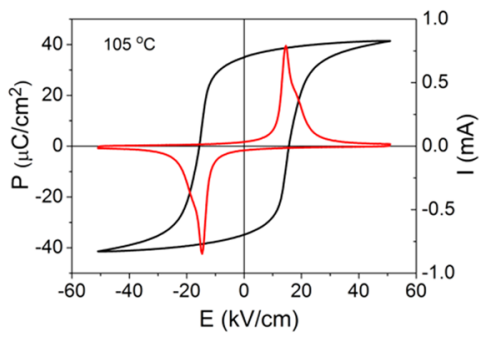

(c)

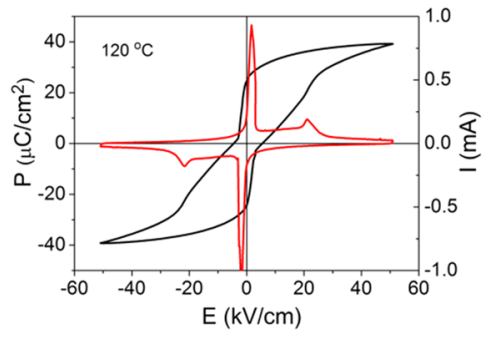

(d)

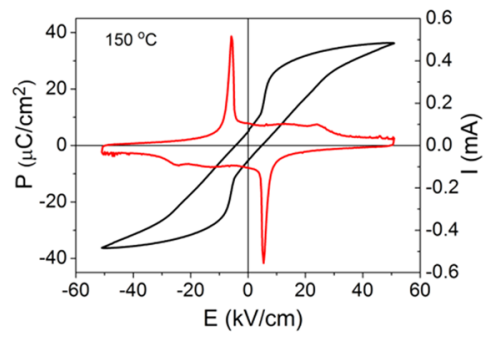

(e)

Figure 6: Polarization and current loops as a function of electric field at a different temperature above RT for d-BNTBT. The appearance of four current peaks at $120^{\circ} \mathrm{C}$ indicates the transition from polar to weak polar phase between 105 and $120^{\circ} \mathrm{C}$. 


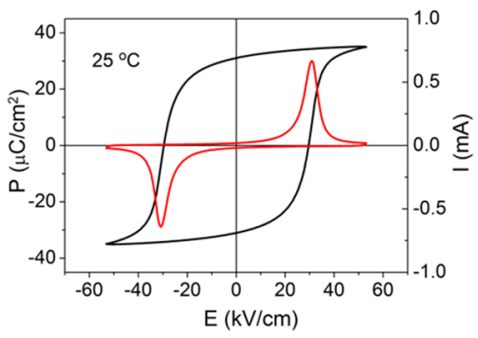

(a)

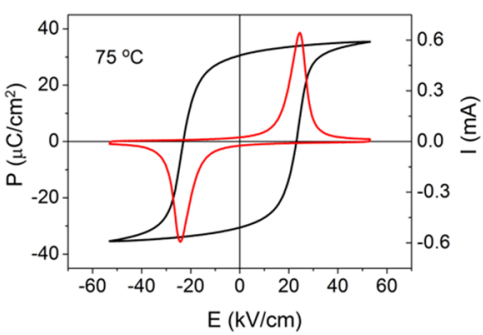

(b)

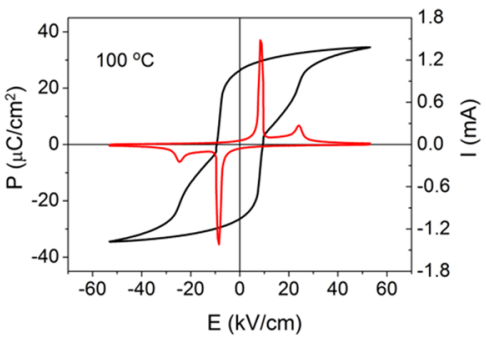

(c)

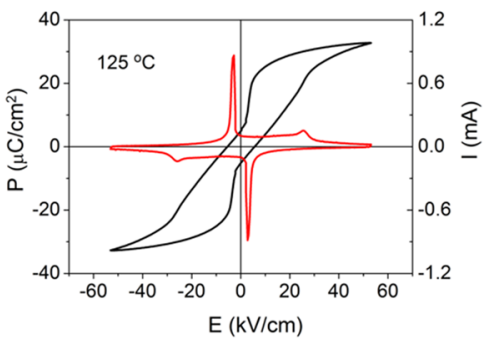

(d)

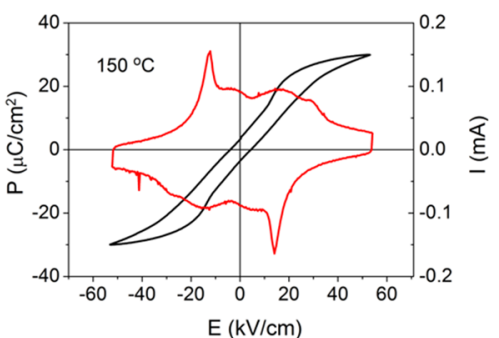

(e)

Figure 7: Polarization and current loops as a function of electric field at a different temperature above RT for ud-BNTBT. The four current peaks observed at $100^{\circ} \mathrm{C}$ much earlier compared to d-BNTBT.

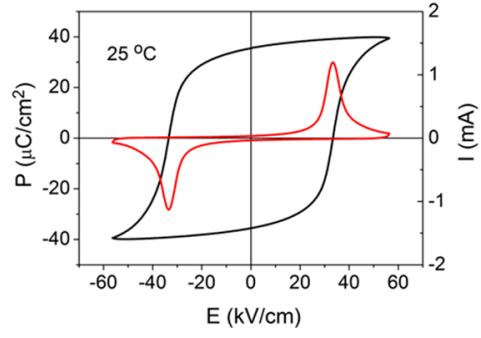

(a)

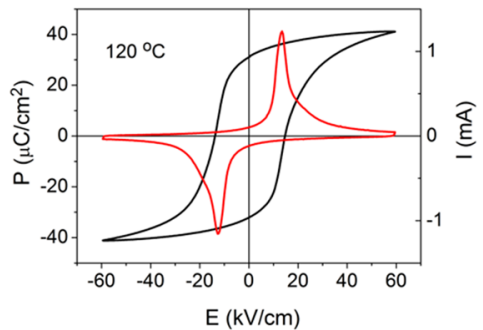

(d)

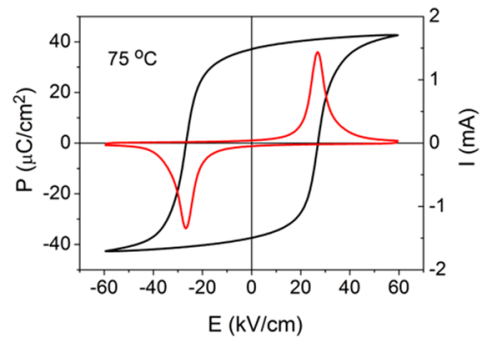

(b)

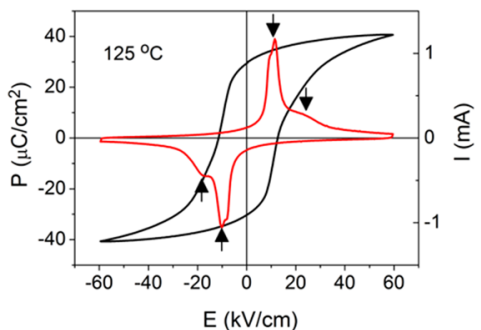

(e)

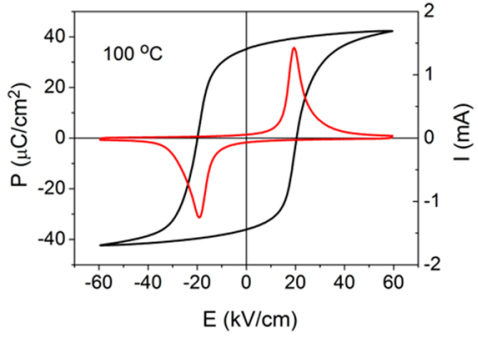

(c)

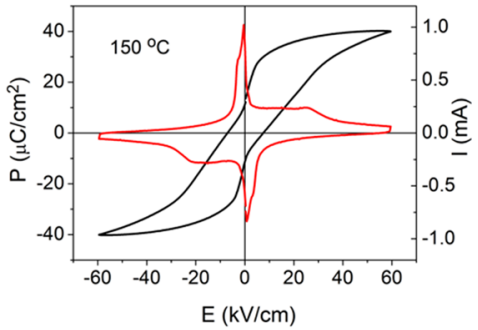

(f)

Figure 8: Polarization and current loops as a function of electric field at a different temperature above RT for lp-BNTBT. The appearance of four current peaks at $125^{\circ} \mathrm{C}$ indicates the transition from polar to weak polar phase between 120 and $125^{\circ} \mathrm{C}$, higher than d-BNTBT and Ip-BNTBT.

depolarization temperature and sharp decrease in $P_{\mathrm{r}}$ measured from dielectric permittivity and $P-I-E$ loops. However, in the case of lp-BNTBT, a significant decrease in $d_{33}$ only occurs above $140^{\circ} \mathrm{C}$, retaining half of its $d_{33}$ at $175^{\circ} \mathrm{C}$. High defect concentration pins the ferroelectric domains and the polar state for lp-BNTBT that persists up to high temperature compared to d-BNTBT and lp-BNTBT. The electrical studies unambiguously rationalize the XRD and Raman findings and illustrate the importance of the processing steps for a highly disordered system like BNTBT and related compounds. 


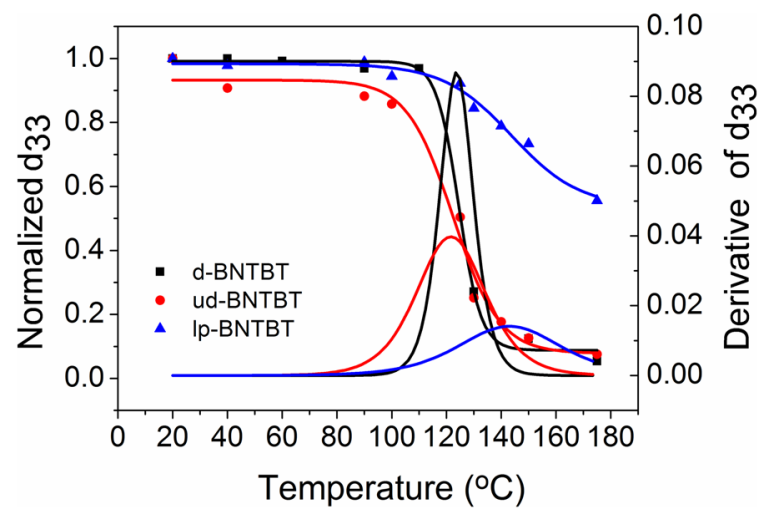

Figure 9: Normalized piezoelectric coefficient $\left(d_{33}\right)$ as a function of temperature d-BNTBT, ud-BNTBT and Ip-BNTBT. The $d_{33}$ profile as a function of temperature corroborates with the $P-I-E$ and dielectric permittivity results.

\section{Conclusions}

A comprehensive study on the effect of processing parameters such as pre-drying of sensitive precursors ( $\mathrm{Bi}$ and $\mathrm{Na}$ ) and their purity on phase and electrical properties of the disordered lead free BNTBT system is presented. The crystal structure evaluated by XRD and Raman shows that a simple step of drying (at a high temperature of the sensitive precursor) can induce a considerable difference in the \% of polar (R3c) and weak polar $(P 4 \mathrm{bm})$ phases. At the nanoscale BNTBT is shows the co-existence of long range ferroelectric domains along with polar nano regions when processed from dried precursors. The weight fraction of polar and weak polar phase and the presence of vacancies and impurity ions results in a decrease in $d_{33}, P_{\mathrm{r}}$ and an increase in $E_{\mathrm{c}}$ (lp-BNTBT) for ud-BNTBT and lp-BNTBT, respectively compared to d-BNTBT. Moreover, modulation in the phase transition temperature was also observed for ud-BNTBT and lp-BNT associated with vacancies and impurity ions on A- or B-sites. This empirical study illustrates the importance of processing for compounds containing sensitive elements like $\mathrm{Bi}$ and $\mathrm{Na}$, which is also relevant for similar compounds.

\section{Experiment}

Three sets of $0.94\left(\mathrm{Bi}_{0.5} \mathrm{Na}_{0.5} \mathrm{TiO}_{3}\right)-0.06 \mathrm{BaTiO}_{3}$ (BNTBT) powders were synthesized by a solid-state reaction method. Among them, two sets of BNTBT powders were obtained from high purity precursors of $\mathrm{Na}_{2} \mathrm{CO}_{3}$ (Sigma, 99.5\%), $\mathrm{Bi}_{2} \mathrm{O}_{3}$ (Alfa Aesar, 99.975\%), $\mathrm{TiO}_{2}$ (Sigma Aldrich, 99.9\%) and $\mathrm{Ba}_{2} \mathrm{CO}_{3}$ (Alfa Aesar, 99.8\%), named as undried BNTBT (ud-BNTBT) and dried BNTBT (d-BNTBT) (Table S1). The ud-BNTBT powders were obtained without any preheating treatment of the precursors, whereas, the d-BNTBT powders were obtained after preheating all the precursors at $\sim 400{ }^{\circ} \mathrm{C}$ for $24 \mathrm{~h}$, before weighing them according to the required composition. The third set of powders were obtained after preheating $\left(\sim 400^{\circ} \mathrm{C}\right.$ for $\left.24 \mathrm{~h}\right)$ of the precursors of $\mathrm{Na}_{2} \mathrm{CO}_{3}$ (Alfa Aesar, 98\%, Lot number 10123239, impurities $\mathrm{NaCl} 0.4 \%, \mathrm{Fe}_{2} \mathrm{O}_{3} \sim 0.003 \%, \mathrm{NaSO}_{4} \sim 0.05 \%$ ), $\mathrm{Bi}_{2} \mathrm{O}_{3}$ (Alfa Aesar, 99\%), $\mathrm{TiO}_{2}$ (Sigma Aldrich, purity 99\%, Lot. SZBF0290V, impurity $\mathrm{As}, \mathrm{Fe}<50 \mathrm{mg} / \mathrm{kg}, \mathrm{Pb}<50 \mathrm{mg} / \mathrm{kg}$ ) and $\mathrm{BaCO}_{3}$ (Alfa Aesar, 99.8\%), having low purity in comparison to the udBNTBT and d-BNTBT powders. This powder set was named as lp-BNTBT. Table S1 shows the precursors details used for the fabrication of all the three sets of samples and details about pre-processing treatments and impurity ions. All of the powders were weighted according to the target composition [0.94(Bi $\left.\left.{ }_{0.5} \mathrm{Na}_{0.5} \mathrm{TiO}_{3}\right)-0.06 \mathrm{BaTiO}_{3}\right]$ and ball milled in Teflon jars containing ethanol media and zirconia balls. The ball milling was performed for $4 \mathrm{~h}$ at $360 \mathrm{rpm}$. After drying the stoichiometric powders in an oven at $100^{\circ} \mathrm{C}$ for $24 \mathrm{~h}$, they were calcined in air at $900{ }^{\circ} \mathrm{C}$ for $2 \mathrm{~h}$ with a heating rate of $5{ }^{\circ} \mathrm{C} / \mathrm{min}$. The calcined powders were ball milled to obtain fine particle size. Bulk pellets for all the ceramics were obtained by uniaxially pressing the powders in a $15 \mathrm{~mm}$ stainless steel die under $\sim 60 \mathrm{MPa}$. The pressed pellets were sintered at $1150{ }^{\circ} \mathrm{C}$ for $4 \mathrm{~h}$ covering them with their respective calcined powders to minimize the loss of $\mathrm{Na}$ and $\mathrm{Bi}$ atoms.

For characterization ud-BNTBT, d-BNTBT and lp-BNTBT pellets were crushed to powders. XRD patterns were collected at room temperature [RT, Panalytical Xpert Pro diffractometer using $\mathrm{Cu}$ Ka radiation $(\lambda=1.5418 \AA)$ ]. Raman spectroscopy was performed on the same powders using a Renishaw spectrometer equipped with $\mathrm{He}-\mathrm{Ne}$ laser source with $(\lambda=631 \mathrm{~nm})$ and optical lens of $\times 50$ at room temperature. The microstructure of a d-BNTBT ceramic sample was studied using a JEOL 2010 transmission electron microscope operated at $200 \mathrm{kV}$ and a FEI Inspect-F scanning electron microscope with an Oxford INCA energy dispersive spectrometer. A TEM lamella was prepared using focused ion beam milling on a FEI Quanta 3D Dual Beam system using a $30 \mathrm{kV} \mathrm{Ga}^{+}$ion beam and currents down to $28 \mathrm{pA}$. When the thickness of the specimen reached about $100 \mathrm{~nm}$, final low-kV cleaning was performed at $5 \mathrm{kV}, 16 \mathrm{pA}$.

The electrical measurements were conducted using a metal-insulator-metal configuration by painting pellets with silver paste (Gwent Electronic Materials Ltd. Pontypool, UK) to produce top and bottom electrodes. The relative permittivity $\left(\varepsilon_{\mathrm{r}}\right)$ and loss tangent $(\tan \delta)$ as a function of temperature (from room temperature to $500^{\circ} \mathrm{C}$ ) were evaluated using an LCR meter (Agilent 4284A, $20 \mathrm{~Hz}$ to $1 \mathrm{MHz}$, Hyogo, Japan) equipped with vertical tube furnace (Lenton, LTF). Current-polarization-electric field $(I-P-E)$ hysteresis loops were obtained at $1 \mathrm{~Hz}$ using hysteresis tester (NPL, UK) in a silicon oil bath from RT to $150^{\circ} \mathrm{C}$. The samples were poled using TREK, HV amplifier. 
Direct piezoelectric coefficient $\left(d_{33}\right)$ was measured using Berlincourt meter at RT (Model ZJ-3B, PIEZO d $\mathrm{d}_{33}$ meter, Institute of Acoustics Academia Sinica). Also, $d_{33}$ was measured at room temperature after subjecting the samples to different temperatures for a holding time of $10 \mathrm{~min}$.

\section{Acknowledgments}

Authors thank for the financial support by EPSRC, MASSIVE Project No. EP/L017695/1. EVR acknowledges FCT, Portugal for the Project I3N, UIDB/50025/2020 and UIDP/50025/2020.

\section{Supplementary information}

The online version contains supplementary material available at https://doi.org/10.1557/s43578-020-00040-1.

\section{Open access}

This article is licensed under a Creative Commons Attribution 4.0 International License, which permits use, sharing, adaptation, distribution and reproduction in any medium or format, as long as you give appropriate credit to the original author(s) and the source, provide a link to the Creative Commons licence, and indicate if changes were made. The images or other third party material in this article are included in the article's Creative Commons licence, unless indicated otherwise in a credit line to the material. If material is not included in the article's Creative Commons licence and your intended use is not permitted by statutory regulation or exceeds the permitted use, you will need to obtain permission directly from the copyright holder. To view a copy of this licence, visit http://creativecommons.org/ licenses/by/4.0/.

\section{References}

1. A.K. Tagantsev, V.O. Sherman, K.F. Astafiev, J. Venkatesh, N. Setter, J. Electroceram. 11, 5-66 (2003)

2. European Union Parliament, European Union Council, Off. J. Eur. Union 46, 19-23 (2003)

3. Y. Saito, H. Takao, T. Tani, T. Nonoyama, K. Takatori, T. Homma, T. Nagaya, M. Nakamura, Nature 432, 84-87 (2004)

4. T. Tadashi, M. Kei-ichi, S. Koichiro, Jpn. J. Appl. Phys. 30, 2236 (1991)

5. W. Jo, J.E. Daniels, J.L. Jones, X. Tan, P.A. Thomas, D. Damjanovic, J. Rödel, J. Appl. Phys. 109, 014110 (2011)

6. R. Garg, B.N. Rao, A. Senyshyn, P.S.R. Krishna, R. Ranjan, Phys. Rev. B 88, 014103 (2013)

7. B. Wylie-van Eerd, D. Damjanovic, N. Klein, N. Setter, J. Trodahl, Phys. Rev. B 82, 104112 (2010)

8. C. Ma, X. Tan, J. Am. Ceram. Soc. 94, 4040-4044 (2011)
9. I.P. Pronin, P.P. Syrnikov, V.A. Isupov, V.M. Egorov, N.V. Zaitseva, Ferroelectrics 25, 395-397 (1980)

10. G.A. Samara, in Solid State Physics, vol. 56, ed. by E. Henry, S. Frans (Academic, San Diego, 2001), pp. 239-458

11. V. Dorcet, G. Trolliard, Acta Mater. 56, 1753-1761 (2008)

12. C. Ma, H. Guo, S.P. Beckman, X. Tan, Phys. Rev. Lett. 109, $107602(2012)$

13. C. Ma, X. Tan, E. Dul'kin, M. Roth, J. Appl. Phys. 108, 104105 (2010)

14. D. Maurya, A. Pramanick, M. Feygenson, J.C. Neuefeind, R.J. Bodnar, S. Priya, J. Mater. Chem. C 2, 8423-8431 (2014)

15. E. Aksel, J.S. Forrester, J.L. Jones, P.A. Thomas, K. Page, M.R. Suchomel, Appl. Phys. Lett. 98, 152901 (2011)

16. V. Dorcet, G. Trolliard, P. Boullay, J. Magn. Magn. Mater. 321, 1758-1761 (2009)

17. S.-T. Zhang, A.B. Kounga, E. Aulbach, Y. Deng, J. Am. Ceram. Soc. 91, 3950-3954 (2008)

18. V. Dorcet, G. Trolliard, P. Boullay, Chem. Mater. 20, 5061-5073 (2008)

19. C. Ma, X. Tan, Solid State Commun. 150, 1497-1500 (2010)

20. S. Uddin, G.-P. Zheng, Y. Iqbal, R. Ubic, J. Yang, J. Appl. Phys. 114, 213519 (2013)

21. P. Patnaik, Handbook of Inorganic Chemicals (McGraw-Hill, New York, 2003).

22. S. Prasertpalichat, D.P. Cann, J. Mater. Sci. 51, 476-486 (2016)

23. I.-T. Seo, S. Steiner, T. Frömling, J. Eur. Ceram. Soc. 37, 1429$1436(2017)$

24. M. Li, M.J. Pietrowski, R.A. De Souza, H. Zhang, I.M. Reaney, S.N. Cook, J.A. Kilner, D.C. Sinclair, Nat. Mater. 13, 31-35 (2014)

25. M. Li, H. Zhang, S.N. Cook, L. Li, J.A. Kilner, I.M. Reaney, D.C. Sinclair, Chem. Mater. 27, 629-634 (2015)

26. C. Hong, Z. Fan, X. Tan, W. Kang, C.W. Ahn, Y. Shin, W. Jo, J. Eur. Ceram. Soc. 38, 5375-5381 (2018)

27. B.N. Rao, A.N. Fitch, R. Ranjan, Phys. Rev. B 87, 060102 (2013)

28. E. Venkata Ramana, F. Figueiras, A. Mahajan, D.M. Tobaldi, B.F.O. Costa, M.P.F. Graca, M.A. Valente, J. Mater. Chem. C 4, 1066-1079 (2016)

29. A. Larson, R. Von Dreele, M. Lujan Jr., GSAS-generalized crystal structure analysis system (Los Alamos National Laboratory, Los Alamos, 1987)

30. D. Rout, K.-S. Moon, V.S. Rao, S.-J.L. Kang, J. Ceram. Soc. Jpn 117, 797-800 (2009)

31. R. Asiaie, W. Zhu, S.A. Akbar, P.K. Dutta, Chem. Mater. 8 226-234 (1996)

32. I. Levin, I.M. Reaney, Adv. Funct. Mater. 22, 3445-3452 (2012)

33. C.A. Randall, D.J. Barber, R.W. Whatmore, J. Mater. Sci. 22, 925-931 (1987)

34. C. Ma, H. Guo, X. Tan, Adv. Funct. Mater. 23, 5261-5266 (2013)

35. S.-Y. Cheng, N.-J. Ho, H.-Y. Lu, J. Am. Ceram. Soc. 89, $2177-$ 2187 (2006) 
36. X.-S. Qiao, X.-M. Chen, H.-L. Lian, W.-T. Chen, J.-P. Zhou, P. Liu, J. Am. Ceram. Soc. 99, 198-205 (2016)

37. W. Jo, S. Schaab, E. Sapper, L.A. Schmitt, H.-J. Kleebe, A.J. Bell, J. Rödel, J. Appl. Phys. 110, 074106 (2011)

38. G. Viola, H. Ning, X. Wei, M. Deluca, A. Adomkevicius, J. Khaliq, M. John Reece, H. Yan, J. Appl. Phys. 114, 014107 (2013)
39. J. Wu, H. Zhang, C.-H. Huang, C.-W. Tseng, N. Meng, V. Koval, Y.-C. Chou, Z. Zhang, H. Yan, Nano Energy 76, 105037 (2020)

40. B.-J. Chu, D.-R. Chen, G.-R. Li, Q.-R. Yin, J. Eur. Ceram. Soc. 22, 2115-2121 (2002) 